\title{
Avant-propos
}

\section{Jacques Gleyse}

\section{OpenEdition}

Journals

Édition électronique

URL : http://journals.openedition.org/trema/1066

DOI : 10.4000/trema.1066

ISSN : 2107-0997

\section{Éditeur}

Faculté d'Éducation de l'université de Montpellier

\section{Édition imprimée}

Date de publication : 1 juin 2010

Pagination : $1-4$

ISSN : 1167-315X

\section{Référence électronique}

Jacques Gleyse, «Avant-propos », Tréma [En ligne], 32 | 2010, mis en ligne le 20 septembre 2010, consulté le 22 septembre 2020. URL : http://journals.openedition.org/trema/1066 ; DOI : https:// doi.org/10.4000/trema.1066

Ce document a été généré automatiquement le 22 septembre 2020.

Trema 


\title{
Avant-propos
}

\author{
Jacques Gleyse
}

1 Il me faut dire ici le plaisir que j'ai eu à coordonner avec Michèle VERDELHAN Bourgade ce numéro de Tréma, consacré à la mixité, au genre et à l'éducation physique en tant qu'indicateur particulier, on pourrait même dire: révélateur, de cette question.

2 Bien que la revue Tréma ait été créée pratiquement à l'ouverture de l'IUFM de Montpellier, en 1991, par Robert Ferras, premier directeur de l'IUFM, Michèle VERDELHAN - BOURGADE, Jacques BERTOUX, Pierre GUIBBERT, Georges ROQUES, Maurice AUPECLE, et le rédacteur de cet éditorial, bien qu'elle soit dotée d'un comité de parrainage institutionnel, d'un conseil scientifique et d'un réseau de correspondants internationaux, bien qu'elle soit également d'une belle facture et d'un beau graphisme, elle n'avait pas encore eu la reconnaissance dont elle bénéficie aujourd'hui. En effet, elle est maintenant partie intégrante de la banque de donnée de revues.org, ce qui est un indicateur de permanence et de qualité pour une revue en sciences humaines. En outre, elle est aujourd'hui présente dans la banque de donnée de l'AERES en sciences de l'éducation et plus généralement en sciences humaines.

3 Le moment de la naissance de Tréma était un moment heureux et un moment de progrès pour la formation des enseignants notamment du second degré. La pédagogie, à la suite du rapport BANCEL et des lois JOSPIN devenait une préoccupation paritaire avec la connaissance savante dans la formation du système secondaire, ce qu'elle 
n'avait jamais été jusqu'alors. L'élève se trouvait placé au centre du système éducatif et les missions de l'enseignant corollairement allaient changer: former, éduquer, enseigner. Bref, les réformes allaient vers une amélioration du système éducatif notamment secondaire en permettant que l'on se préoccupe davantage des élèves en difficulté et surtout que l'on enseigne pour le plus grand nombre et pas seulement pour quelques privilégiés (les garçons l'étaient, statistiquement, à ce moment là). Évidemment dans cette dynamique la mixité ne pouvait, en aucun cas, être remise en cause puisque l'on pensait massivement (à part quelques réactionnaires notoires) qu'elle était l'un des éléments qui permettait aux élèves de chaque sexe de s'enrichir mutuellement.

4 C'est évidemment dans un tout autre contexte que j'écris cet avant-propos. La mixité a récemment été remise en cause par quelques écrits médiatisés. Un rapport du Sénat a rediscuté sur le fond cette question. Les IUFM ont été intégrés dans une université en perdant de ce fait une partie de leur originalité et de leur autonomie. La pédagogie a été vouée aux gémonies par des ouvrages à grande diffusion et très médiatisés. La télévision tout comme le cinéma nous ont renvoyés récemment à des modèles pédagogiques caricaturaux, éculés voire obsolètes, sans aucune pertinence dans les sociétés contemporaines. Pourtant, quelques obstinés continuent à chuchoter les noms d'Ovide DECROLY, Maria MONTESSORI, John DEWEY, Célestin FREINET, Roger COUSINET, Karl ROGERS, A.S. NEIL, Fernand OURY, Aïda VASQUEZ, Jacques PAIN, Catherine POCHET, Yvan ILLICH, Fernand DELIGNY, René LAFFITTE (je dois en oublier beaucoup), mais leur nombre est en diminution constante. Bref, comme dans un renversement incongru, le progrès humain et pédagogique passerait par la régression. Le mot réforme est devenu, lui-même, synonyme de régression humaine et parfois pédagogique. Dans ce contexte, on comprend que démixer les classes ou renoncer à la coéducation ait paradoxalement pu être considéré comme un progrès.

Dans ce contexte donc, le numéro permet de poser à nouveau le problème de cette pratique dans l'ensemble de l'école. L'originalité, ici consistera d'une part à s'interroger sur les apports de la mixité scolaire à l'égalité filles - garçons, d'autre part à examiner plus particulièrement la mixité dans cet espace spécifique qu'est l'éducation physique et sportive, champ se situant, en définitive, entre deux institutions (les Écoles et le Sport). En cela, l'éducation physique peut être un parfait analyseur des stéréotypes sociaux et scolaires pour ce qui est de la coéducation ou de la mixité.

On se souvient de l'ouvrage de Nicole MOSCONI La Mixité dans l'enseignement secondaire. Un faux semblant, qui posait bien la différence entre coéducation et mixité, montrant notamment, en 1989, que le système éducatif secondaire était fondé sur un « masculin neutre ", un peu comme le "deuxième sexe » de Simone DE BEAUVOIR (1949) était un "produit intermédiaire entre le mâle et le castrat». En éducation physique Pierre ARNAUD (1996) constatait, lui aussi, que l'éducation physique féminine était une "éducation physique masculine atténuée ».

De toute évidence, une véritable mixité scolaire, n'est pas une coéducation, il ne s'agit pas seulement de mettre en présence les deux sexes dans le processus éducatif mais de faire en sorte que ceux-ci s'enrichissent mutuellement. On pourrait reprendre d'ailleurs là l'un des adages du Judo traditionnel : «Entraide et prospérité mutuelle ». En cela, contrairement à la coéducation, la mixité, spécificité française, serait une méthode pédagogique aussi bien qu'une utopie ou qu'un idéal à atteindre. 
8 Les articles qui suivent, que je suis heureux d'introduire ici et pour lesquels je voudrais remercier vivement les auteurs, ne posent pas tous cette question mais, en tout état de cause, contribuent à alimenter ce débat, dans lequel la formation des enseignants tient une grande place.

9 Le texte d'Yveline FUMAT, professeure des universités honoraire, en sciences de l'éducation, qui fut l'une des créatrices de son champ de prédilection à l'Université Paul VALERY et qui a réalisé de nombreux travaux sur les questions de mixité et de genre, pose la question de l'éducation mixte dans la famille et à l'école. Ainsi ne se limite-t-elle pas au champ scolaire et permet-elle d'ouvrir un débat dans un espace plus large.

Dominique GAUTHIER-RIEUCAU, personnel de direction, professeur d'histoire géographie, qui a réalisé des formations sur le genre à l'IUFM de Montpellier et mis en œuvre plusieurs colloques sur le thème, évoque la question de la formation à la mixité et plus généralement des formations à la mixité scolaire, en la faisant passer au filtre de la question du genre. Elle permet notamment d'éclairer de manière originale cette question en posant la question centrale de l'égalité - altérité, retrouvant ainsi l'idée d'une mixité égalitaire mais aussi enrichissement mutuel.

11 Bénédicte GENDRON, professeure en sciences de l'éducation, dont les travaux reconnus ont porté sur les compétences émotionnelles dans le monde laborieux, montre la complémentarité des capitaux émotionnels des filles et des garçons et notamment, l'immense utilité des compétences émotionnelles féminines dans certains domaines particuliers mais aussi leur spécificité.

12 Karine ISABELLE, conseillère principale d'éducation, qui a réalisé un mémoire de Master 2 de sciences de l'éducation sur les questions de stéréotypes de genre en grande section de maternelle, étude originale car recherche-action fondée sur un travail ethnographique de longue durée (un an et demi), fait apparaître des programmes cachés d'éducation chez deux enseignants d'école maternelle et examine les stéréotypes de genre dans les jeux des élèves en cour de récréation.

13 Pour ma part, je réalise, en le confrontant aux conclusions du rapport du Sénat de 2003, un essai d'histoire de la mixité dans l'éducation et en éducation physique et sportive, en tentant de montrer à la fois les consonances des deux champs ainsi que leurs dissonances. On voit là que le sport et la mixité ne font pas bon ménage. Ce texte permet aussi de réfuter quelques idées reçues sur la question d'une apparition récente de la mixité qui confond souvent les textes officiels et la réalité du terrain et confond aussi enseignement du second degré et enseignement secondaire.

14 Geneviève COGERINO, professeure en STAPS et chercheuse au CRIS de Lyon, réalise des travaux depuis un certain nombre d'années et dirige plusieurs thèses sur les questions de genre et de mixité en éducation physique. Elle a également dirigé et publié de nombreux textes sur ce sujet. Elle pose là la question du modèle corporel formalisé et privilégié par les enseignantes d'éducation physique en même temps que des contradictions ou des tensions qui peuvent exister entre un modèle corporel sportif et un modèle corporel féminin.

15 Enfin, le texte de notre collègue brésilienne Helena ALTMANN, professeure à la faculté d'Unicamp (Sao Paolo, Brésil) montre comment l'introduction de la mixité en éducation physique au Brésil, depuis les années 1990, est un véritable défi à relever pour l'institution et pour les enseignants eux-mêmes. En nous faisant revenir un peu en arrière dans le temps elle nous permet de saisir la complexité du problème notamment 
en montrant comment les travaux sur le genre transforment le regard porté sur la mixité et sur la pratique de l'éducation physique.

Loin d'être dépassé, ce thème de la mixité paraît toujours d'actualité et toujours extrêmement riche lorsqu'il est question de formation des enseignants mais aussi de pratiques pédagogiques innovantes. L'équipe de recherche Santesih (J.E. 2516), dont je suis l'un des animateurs, s'intéressant à la question des vulnérabilités et des "situations de handicaps", est particulièrement sensible aux questions du genre dans l'éducation et les pratiques sociales. Elle encadre de nombreux travaux d'étudiants dans ce domaine. Elle soutient donc tout naturellement ce type d'initiatives et de publications. Je souhaite que le document proposé aux lecteurs puisse contribuer à éclairer un peu les pratiques d'interventions mais aussi la réflexion théorique sur le sujet.

17 Castries le 11 Mai 2010.

\section{AUTEUR}

\section{JACQUES GLEYSE}

Professeur des universités, STAPS, Montpellier, docteur en Sciences de l'Éducation 